\title{
Development of Point-of-care Paper Based Strip for the Detection of Simple Antipyretic-analgesic Drugs
}

\author{
GVSR Pavan Kumar*, K Srinivasa Rao
}

Department of Chemistry, MVGR College of Engineering, Vijayaramnagar Campus, Chintalavalasa, Vizianagaram, Andhra Pradesh, INDIA.

\begin{abstract}
A paper-based strip was developed by the authors for the detection and quantitative determination of paracetamol in bulk and pharmaceutical formulations for point-of-care testing prior to the assay prescribed in the respective pharmacopeia. In the present paper, lab-on-chip technology was used for the assay of a simple NSAID drug paracetamol. The qualitative analysis of the drug on the strip was performed by using three different reagents such as sodium Meta vanadate $(1 \%)$, potassium dichromate $(0.01 \mathrm{M})$, N-bromosuccinamide $(1 \%)$. This method employs dissolution of the powdered sample of drug in distilled water, followed by placing the sample solution of the drug on the strip of paper which was previously impregnated with the reagent. Drug concentrations were quantified from color intensity images using a scanner in conjunction with image processing software MATLAB. Linear calibration curves were generated for the drug with each of the reagent. The results obtained were compared with standard methods such HPLC and found to be in accordance with standards. This technology provides qualitative analysis and a prior easy step before assay of the drug at appreciably reduced costs.
\end{abstract}

Key words: Pharmaceutical formulations, Paracetamol, Point-of-care devices, Wax printing, MATLAB.

\section{INTRODUCTION}

According to the World Health Organization, diagnostic devices for developing countries should be assured; affordable, sensitive, specific, user-friendly, rapid and robust, equipment free and deliverable to end users. ${ }^{1}$ In a paper-based strip device, the flow is driven by capillary forces. These require only small volumes of fluid and little or no external supporting equipment or power because fluid movement in such devices is controlled largely by capillary and evaporation. ${ }^{2}$ These devices were widely used in the analysis of glucose, protein, diagnostics and bioassays. and in inorganic metal analysis. ${ }^{3-11}$ Most of the qualitative and quantitative analytical procedures employed for the assay of pharmaceutical formulations involve sophisticated, highly expensive instruments such as chromatographic and spectrophotometric technologies with perfect accuracy. Our present research is aimed at the detection and assay of the chosen drug prior to chromatographic analysis, using a paper based strip. The same was found to be unreported in literature so far. Hence a lab-on-chip technology was proposed by the authors for the qualitative and quantitative analysis of the selected drug. The higher intake of the drug causes liver damage. ${ }^{12}$ Hence its assay by a sensitive, simple and economically feasible method is important. All of these were achieved by the present method proposed by the authors. In this method drug concentrations were quantified from color intensity images using a scanner in conjunction digital image processing software in MATLAB. ${ }^{13}$ This method is found to be advantageous when and where an immediate and on the spot testing of the drug is required. Such strips are helpful for drug inspecting authorities, clinical analysts and useful for the consumer when its quality is to be determined. Most of the analysts do not possess knowledge regarding the use of sophisticated equipment. Hence such a strip accommodates rapid, easy, simple, cheap and eco-friendly method for the qualitative and quantitative analysis of the drug chosen that can be conducted by a person who is not well versed with the science and technology
DOI: 10.5530/ijopp.12.4.48

Address for correspondence: GVSR Pavan Kumar, Department of Chemistry, MVGR College of Engineering, Vijayaramnagar Campus, Chintalavalasa,

Vizianagaram-535005, Andhra Pradesh, INDIA.

Phone no: +919989206996

Email Id: prs_ganti@yahoo.co.in

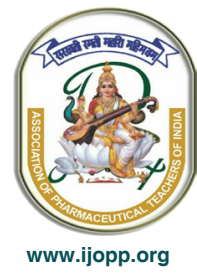


behind. Three different reagents were used in the present study for the development of paper strips, to study the effectiveness of the reaction with the drug.

\section{MATERIALS AND METHODS}

\section{Experimental}

All the chemicals used were of Anal $\mathrm{R}$ grade. Double distilled water was used throughout the experimentation. ELICO LI $120 \mathrm{pH}$ meter was used for $\mathrm{pH}$ measurements. MATLAB software was used for the digital color analysis.

\section{Standard solutions}

Pure sample of paracetamol was synthesized in the laboratory. A solution of such sample was prepared by dissolving an adequately weighed quantity of the drug in distilled water. Such solution is filtered and the filtrate solution was standardized. ${ }^{14}$

Reagent A solution was prepared by dissolving $1 \mathrm{~g}$ of sodium meta vanadate in $100 \mathrm{~mL}$ of distilled water and after complete dissolution the solution was made up to the mark.

Reagent B solution was prepared by dissolving $0.49 \mathrm{~g}$ of potassium dichromate in $100 \mathrm{~mL}$ of distilled water and after complete dissolution the solution was made up to the mark.

Reagent $\mathrm{C}$ solution was prepared by dissolving $1 \mathrm{~g}$ of $\mathrm{N}$-Bromosuccinamide in $100 \mathrm{~mL}$ of distilled water and after complete dissolution the solution was made up to the mark.

\section{Preparation of the strips for qualitative and quantitative analysis}

Whatman No. 1 paper was chosen for the preparation for the paper strip. Strips of the paper with dimensions were cut and dipped in each of the reagent solutions. After a predetermined time these paper strips were removed from the reagent trough and dried to room temperature. Such strips were sandwiched between two thick transparent sheets and laminated. The strip was pierced at the center for the introduction of the drug sample solution.

\section{Quantitative image processing}

Images of the paper devices were scanned ${ }^{15}$ and stored in JPEG format at 300dpi. And the images of the spots were analyzed by MATLAB for finding their R, G and $B$ values from which the values of $H, S$ and $V$ were calculated. The RGB color model is an additive color model in which red, green and blue light are added in various ways to produce a broad array of colors. Any color can be analyzed to obtain its corresponding R, G and $B$ value. The effective intensity for any color values of color spots was calculated as follows

$A_{r}=-\log \left(R_{s} / R_{b}\right)$

$A_{g}=-\log \left(G_{s} / G_{b}\right)$

$\mathrm{A}_{\mathrm{b}}=-\log \left(\mathrm{B}_{\mathrm{s}} / \mathrm{B}_{\mathrm{b}}\right)$

Where $A_{r}, A_{g}$ and $A_{b}$ are effective intensities of the red, green and blue color respectively. $R_{s}, G_{s}, B_{s}$ and $R_{b}, G_{b}$, $B_{b}$ refer to $R, G$ and $B$ values of the sample and blank respectively.

The hue-oriented color space completely describes a color by means of three different components: hue $(\mathrm{H})$, saturation $(\mathrm{S})$ and value $(\mathrm{V})$. These values can be calculated by using the following equations. ${ }^{15}$

$$
H=60^{\circ} \mathrm{X} \quad\left\{\begin{array}{l}
\text { Undefined if } \mathrm{C}=0 \\
(\mathrm{G}-\mathrm{B} / \mathrm{C}) / 6, \text { if } \mathrm{M}=\mathrm{R} \\
(\mathrm{B}-\mathrm{R} / \mathrm{C})+2) / 6, \text { if } \mathrm{M}=\mathrm{G} ;((\mathrm{R}-\mathrm{G} / \mathrm{C}+4) / \\
\\
\text { 6, if } \mathrm{M}=\mathrm{B}
\end{array}\right.
$$

Where $\mathrm{C}=\mathrm{M}-\mathrm{m} \quad\left\{\begin{array}{l}\mathrm{M}=\max (\mathrm{R}, \mathrm{g} \text { and } \mathrm{B}) \\ \mathrm{m}=\min (\mathrm{R}, \mathrm{g} \text { and } \mathrm{B})\end{array}\right.$

$\mathrm{S}=0$ if $\mathrm{C}=0$ and $\mathrm{S}=\mathrm{C} / \mathrm{V}$ otherwise

$\mathrm{V}=\max (\mathrm{R}, \mathrm{G}, \mathrm{B})$

Using these formulae and MATLAB software, the scanned images were analyzed and $\mathrm{H}, \mathrm{S}$ and $\mathrm{V}$ values of the same were calculated.

\section{RESULTS AND DISCUSSION}

The three reagents A, B and C, used for the detection and determination of paracetamol are oxidizing agents. All the three reagents were reduced by the drug and exhibited the same color, deep violet blue. Paper strips impregnated with the reagents, responded in the same manner and exhibited deep violet blue color (Figure 1) as and when the drug sample solution is introduced on the strip. Reagent A and C are colorless by themselves. Reagent $\mathrm{B}$ is a pale yellow color solution, when the strip made ready by impregnating the reagent and after drying it become paler by making easy for the color detection after the introduction of the drug on to it. Calibration curves for the drug and the three different reagents were drawn for concentration of the drug and $H$ values obtained. The calibration curve was found to be linear 
for 2.4-9.6 $\mu \mathrm{gmL}^{-1}$ with reagent A, B and C (Figure 2-4). Correlation factor from the calibration curves of drug with reagent $\mathrm{A}, \mathrm{B}$ and $\mathrm{C}$ were found to be $0.973,0.969$ and 0.969 respectively. These values were well within the prescribed standard limits. Commercially available tablets of paracetamol of different pharmaceutical companies were analyzed by the method proposed. Ten tablets of each of the company were ground to a fine powder and made a solution..$^{13}$ One micro litre of such solution is introduced on the paper based strip and from the R, G and $B$ values and $H$ value calibration curve; concentration of the drug can be calculated. From the results obtained, RSD for the analysis with the reagents was found to be $1.92 \%, 1.86 \%$ and $1.82 \%$ respectively. These values were well within the prescribed standards. A comparative table for the measured and actual value was presented in Table 1. The results obtained in the method proposed were compared with HPLC technology and found to be accurate. Table 2 gives a comparison between HPLC and paper strip methods.

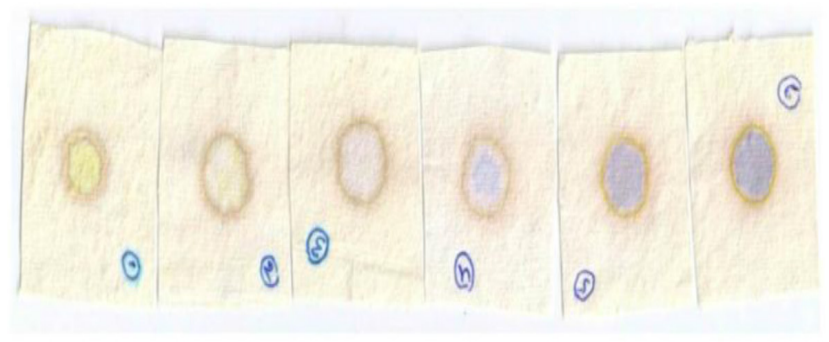

Figure 1: Detection of paracetamol using the paper-based strip developed.

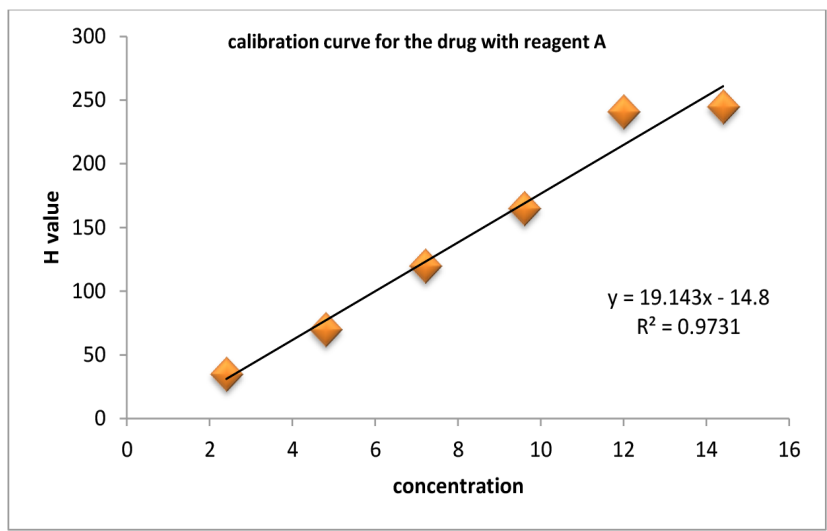

Figure 2: Calibration curve for the drug with reagent $A$.

\section{Optimization conditions}

Injection volume: The influence of volume of analyte which must be injected onto the paper strips was investigated. The optimum sample volume was found to be $1 \mu \mathrm{L}$. An increased volume injection leads to ring dispersal and decrease in intensity.

\section{Effect of reagent concentration}

An optimum concentration of $1 \%$ was fixed for reagent $\mathrm{A}$ and $\mathrm{C} ; 0.49 \%$ or $0.01 \mathrm{M}$ was fixed as optimum concentration for reagent $\mathrm{B}$. Above and below these concentration levels the color produced was found to be unstable and hence these were fixed as optimum concentration.

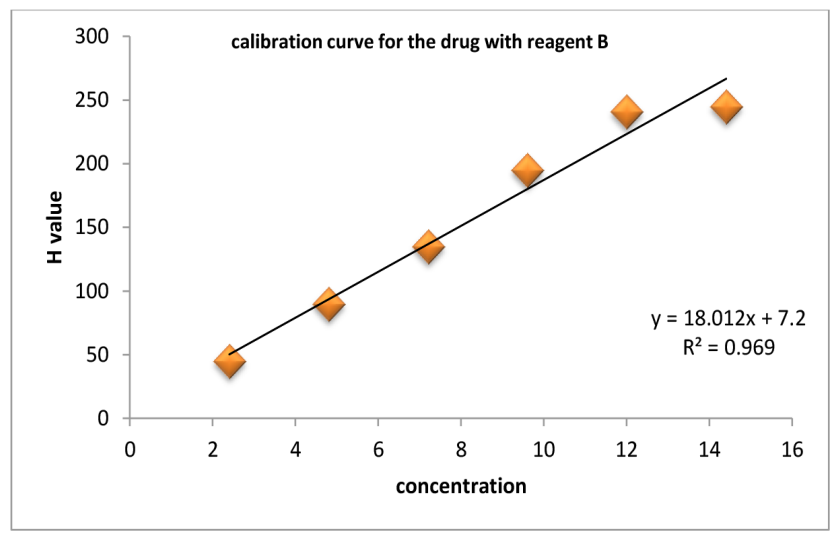

Figure 3: Calibration curve for the drug with reagent B.

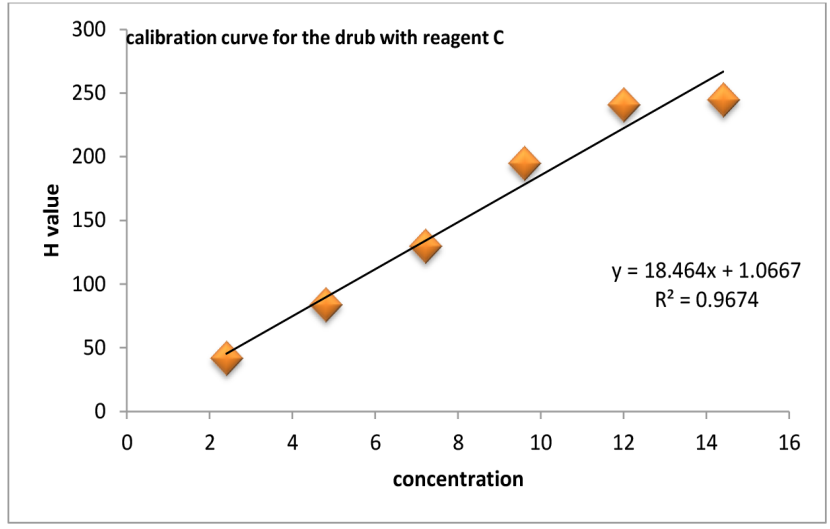

Figure 4: Calibration curve for the drug with reagent $\mathrm{C}$.

\begin{tabular}{cccccccccccc}
\multicolumn{3}{l}{ Table 1: Real sample analysis report. } & \multicolumn{3}{c}{ Reagent A } & \multicolumn{3}{c}{ Reagent B } & \multicolumn{3}{c}{ Reagent C } \\
\cline { 2 - 11 } S.No & $\begin{array}{c}\text { Name of the } \\
\text { drug }\end{array}$ & PM & SM & RSD & PM & SM & RSD & PM & SM & RSD \\
\hline 1 & Paracetamol & 99.8 & 99.9 & 1.92 & 99.7 & 99.9 & 1.86 & 99.6 & 99.9 & 1.82 \\
2 & Calpol & 98.6 & 98.9 & 1.91 & 98.7 & 98.9 & 1.84 & 98.4 & 98.9 & 1.8 \\
3 & Saidol P & 99.2 & 99.4 & 1.92 & 99.3 & 99.4 & 1.86 & 99.2 & 99.4 & 1.82 \\
\hline
\end{tabular}

PM- proposed method, SM- standard method; RSD- relative standard deviation 
Table 2: Comparison between HPLC and paper strip methods.

\begin{tabular}{ccccc}
\hline S.No & $\begin{array}{c}\text { Concentration } \\
\text { of the drug } \mathbf{~ n g} \\
\text { ml }^{-1}\end{array}$ & $\begin{array}{c}\text { Paper strip } \\
\text { method }\end{array}$ & $\begin{array}{c}\text { HPLC } \\
\text { method }\end{array}$ & $\begin{array}{c}\% \\
\text { error }\end{array}$ \\
\hline 1 & 10 & 9.9 & 10 & 1 \\
2 & 15 & 15.2 & 15 & 1.33 \\
3 & 20 & 19.9 & 20 & 1 \\
4 & 25 & 24.8 & 25 & 1.3 \\
\hline
\end{tabular}

\section{Drying methods}

After the impregnation of the reagents on the paper based strip, these are need to be dried. Generally employed methods for drying include, drying at room temperature, in an oven and hot air oven. Considerable changes in signals were not observed when different methods of drying were employed. Hence it was proposed to dry the paper based device at room temperature to avoid undesired results.

\section{Interference studies}

Interference studies were carried out with excipients such as starch, lactose, magnesium stearate and sorbitol etc. It was found that these excipients have no interference in the assay of the drug using the strip developed.

\section{CONCLUSION}

The strip developed is an inexpensive way for the detection and quantitative determination prior to chromatographic assay of the drug. This method provides a simple, rapid, safe way for the assay of the drug. Moreover, with the existence of colorimetric assays for the drug, the strip can be fitted for specific work environments. The proposed device and method was useful for drug inspection, pharmaceutical industries for the detection of the drug during research and development, synthesis, production and quality control activities.

\section{ACKNOWLEDGEMENT}

The authors thank the Principal and management of MVGR College of Engineering (Autonomous), Vizianagaram-535005, India for the facilities provided and their constant support and encouragement.

\section{CONFLICT OF INTEREST}

The authors declare that there are no conflicts of interest.

\section{ABBREVIATIONS}

$\mathbf{A}_{\mathrm{b}}$ : Effective intensity of blue; $\mathbf{A}_{\mathrm{g}}$ : Effective intensity of green; $\mathbf{A}_{\mathbf{r}}$ : Effective intensity of red; B: Blue; G: Green; H: Hue parameter; HPLC: High Performnace Liquid Chromatography; R: Red; $\mathbf{R}_{b}, \mathbf{G}_{\mathrm{b}}, \mathbf{B}_{\mathbf{b}}$ : RGB values for blank; $\mathbf{R}_{\mathrm{s}}, \mathbf{G}_{\mathrm{s}}, \mathbf{B}$ : RGB Values for sample; S: Saturation; V: Value.

\section{REFERENCES}

1. Preeling RW, Holmes KK, Mabey D, Ronald. Rapid tests for sexually transmitted diseases. Sex Transm Infect. 2006;82(suppl 5):v1-6.

2. Martinez AW. Diagnostics for the developing world. Anal Chem. 2010;82(1):3-10.

3. Mentele MM, Josephine C, Kristean K, John V. Micro fluidic based analytical device for particulate matter. Anal Chem. 2012;84(10):4474-80.

4. Vald O, Dell D, David E. Smart phone based health accessory for colorimetric detection of biomarkers in sweat and saliva. Lab on Chip. 2013;13(16):3232-8.

5. Kricka LJ, Sia SK. Part of a special issue on point of care Micro fluidic diagnostics. Lab on Chip. 2008;8(12):1988-91.

6. Yetisen AK, Akram MS, Christopher RL. Paper based micro fluidic point of care diagnostic devices. Lab on Chip. 2013;13(12):2210-51.

7. Martinez AW, Philips ST, Emanuel C, Thomas SW III, Hayat S, George MW. Simple telemedicine for developing regions, camera phones and paper based Microfluidic devices for real time, off site diagnosis. Anal Chem. 2008;80(10):3699-707.

8. Nuria LR, Vincenzo FC, Miguel ME, Fernando BL, Dermot D, Alberto JP, et al. Smart phone based simultaneous $\mathrm{pH}$ and Nitrite colorimetric determination for paper micro fluidic devices. Anal Chem. 2014;86(19):9554-62.

9. Sia SK, George MW. Microfluidic devices fabricated in poly (dimethyl siloxane) for biological studies. Electrophoresis. 2003;24(21):3563-76.

10. Paul Y, Thayne E, Elainfu, KH, Milton RT, Bernhard HW. Microfluidic diagnostic technologies for global public health. Nature. 2006;442(7101):412-8.

11. Yao L, Wei WS, Lu J, Qin J, Lin B. Rapid photo typing of paper based micro fluidics with was for low cost, portable bioassays. Electrophoresis. 2009;30(9):1497-500.

12. Pavan KGVSR, Ramana $P$, Sravanthi $N$ and Sreerama Murty B. Detection and Spectrophotometric determination of paracetamol using $V(V)$. Journal of Indian Chemical Society. 2011;86(9):893-5.

13. Raquib B, Soumyajit R, Parthin M, Subhajit B, Abhishek M, Sauvik DG. MATLAB based image editing and color detection. International Journal of Scientific and Research Publications. 2014;4(1):1-6

14. Carriho E, Martinez AW, Whitesides GM. Understanding Wax Printing: A Simple Micro-patterning Process for Paper-Based Microfluidics. Anal Chem. 2009;81(16):7091-5.

15. Capitan-Vallvey LF, Ruiz NL, Martinez-OImos A, Miguel ME, Alberto JP. Recent developments in computer vision-based analytical chemistry: A tutorial review. Analytica Chimica Acta. 2015;899:23-56. 